\title{
Miradas Críticas sobre la Intervención Educativa en Diversidad Sexual
}

\section{Critical Views on Educational Intervention in Sexual Diversity}

\author{
Caterine Galaz * \\ Lelya Troncoso \\ Rodolfo Morrison \\ Universidad de Chile
}

\begin{abstract}
Para reflexionar sobre los modos en los cuales el género y la sexualidad se han visibilizado en el ámbito educativo es importante reconocer que, aun cuando no se abordan explícitamente, su presencia es transversal: están en todas partes y "toda educación es sexual”. Se trata de una realidad paradójica en la que, tal como ya ha problematizado Foucault, pareciera tratarse de un tema tabú que sin ser abordado directamente forma parte de discursos omnipresentes. En este artículo buscamos interrogar los modos en que se ha visibilizado la diversidad sexual en el ámbito educativo (en políticas, discursos e intervención directa); sobre los efectos que tienen estas actuaciones, y si éstas posibilitan una lectura crítica a la heteronormatividad y a la lógica binaria de comprensión de las relaciones de género. Lo hacemos poniendo en cuestión, desde una mirada feminista, algunos elementos heterosexistas y normalizantes que consideramos aún presentes en los discursos educativos vigentes.
\end{abstract}

Descriptores: Heteronormatividad, Género, Diversidad sexual, LGTBI, Políticas educativas.

In order to reflect on the ways in which gender and sexuality have been made visible in the educational realm it is important to recognize that, even if these issues are not explicitly addressed, their presence is transversal, they are everywhere and "all education is sexual". This is a paradoxical situation, as it has been already problematized by Foucault, in which it seems to be a taboo subject that is not dealt with directly, but has a ubiquitous discursive presence. In this article we seek to interrogate the ways in which sexual diversity has been made visible in the educational realm (in policies, speeches and direct intervention); the effects of these actions, and if they allow a critique of heteronormativity and the binary understanding of gender relations. From a feminism perspective, we seek to question some heterosexist and normalizing elements that we consider to be still present in current educational speeches.

Keywords: Heteronormativity, Gender, Sexual diversity, LGBTI, Education policies. 


\section{Introducción}

La demanda por una "educación no sexista" se ha ido instalando con fuerza desde parte del movimiento estudiantil en Chile ${ }^{1}$. ¿Pero qué se entiende por educación no sexista? Las respuestas parecen ser más bien complejas, múltiples y abiertas. No es nuestra intención dar una resolución definitiva a dichas interrogantes en este artículo, más bien nos motiva aportar a ampliar y complejizar el debate en torno a las intervenciones educativas relacionadas con la diversidad sexual en Chile desde una perspectiva feminista.

Tal como afirma el Centro Latinoamericano de Sexualidad y Derechos Humanos:

La formulación de leyes contra la discriminación no basta para hacer cesar la
violencia en las relaciones de género y la intolerancia con respecto a las diferentes
orientaciones sexuales e identidades de género. Es fundamental apuntar a la
transformación de la cultura, las mentalidades y las prácticas sociales. (Valdés et
al., 2011, p. 7)

Consideramos que las propuestas feministas son centrales en las transformaciones sociales más profundas que permiten generar cambios concretos y significativos en nuestros modos de construir realidades y relaciones generizadas.

Por ello, en este artículo buscamos interrogar sobre los modos en que se ha incorporado y visibilizado la diversidad sexual en el ámbito educativo (a nivel de políticas, discursos e intervención directa); sobre los efectos que tienen estas políticas e intervenciones educativas en cuanto a la diversidad sexual, y si estas actuaciones posibilitan una crítica a la heteronormatividad y a la lógica binaria de comprensión de las relaciones de género.

Para reflexionar en torno a los modos en los cuales el género y la sexualidad se han incorporado y visibilizado en el ámbito educativo es importante reconocer que, aun cuando no se abordan explícitamente, su presencia es transversal: están en todas partes y toda educación es sexual (Morgade, 2011). Se trata por lo tanto de una realidad paradójica en la que, tal como ya ha problematizado Foucault (1977), pareciera tratarse de un tema tabú que sin ser abordado directamente forma parte de discursos omnipresentes.

En el caso de Chile, no existe una Ley de educación sexual integral, sino una Ley $\mathrm{N}^{\circ}$ 20.418, sobre información, orientación y prestaciones en materia de regulación de la fertilidad (MINSAL, 2010), a partir de la cual se instala desde el año 2010 la obligatoriedad de educación sexual en la enseñanza media chilena. Sin embargo, al centrarse en la regulación de la fertilidad se limita lo que se entiende por educación sexual desde una perspectiva heterosexista y medicalista centrada en derechos y salud reproductiva. Fue el Ministerio de Educación el encargado de apoyar la promoción de programas de educación sexual, cuyas principales orientaciones fueron el pluralismo y la libre elección, para facilitar así al sistema educacional una abanico de propuestas ideológicamente diversas (de cristianos a laicos) que asegurara la libertad de enseñanza y el derecho preferente de los padres (Palma, Reyes y Moreno, 2013). En la comisión de profesionales que seleccionaron los siete programas de educación sexual no participaron

\footnotetext{
${ }^{1}$ Durante el año 2014 se realizó el Primer Congreso por una Educación no sexista, convocado por la Federación de Estudiantes de la Universidad de Chile $(\mathrm{FECH})$ como coralario de varias reflexiones previas sobre el sexismo en los diferentes niveles educativos en el país. http://www.eldesconcierto.cl/pais-desconcertado/movimientossociales/2014/09/12/por-que-es-necesaria-una-educacion-sexista-en-chile/
} 
expertos del campo de la educación y ningún/a experto/a en temas de género o sexualidad ${ }^{2}$.

La ausencia de una mirada crítica de género y de perspectivas feministas para re/pensar la educación sexual en Chile facilita la reproducción de miradas reduccionistas que significan la sexualidad como "natural", limitándose ésta a la dotación biológica y fisiológica del sexo, perdiéndose de vista la dimensión histórica de los cuerpos sexuados que son a su vez afectados por determinaciones sociales, de género, económicas, religiosas, generacionales, de capacidad y étnicas, entre otras, las cuales van a dar forma a los modos en los cuales las personas viven su sexualidad (Morgade, 2006).

En esta línea, las intervenciones educativas forman parte de procesos de construcción de subjetividades y de relaciones sexuadas y generizadas, siendo por lo tanto ámbitos necesarios de problematizar desde una mirada feminista que busca aportar a la transformación social de un orden tradicional, binario y opresor del sexo/género. Nos ubicamos desde el campo de las teorizaciones feministas, las que se han dedicado a debatir en torno a las conceptualizaciones y relaciones entre sexo, género y sexualidad, abordando sus dimensiones epistemológicas, políticas, históricas, sociales, económicas y materiales (Dorlin, 2009). Nos interesa especialmente problematizar de qué modo está operando la noción de diversidad en el ámbito educativo y cómo determinadas conceptualizaciones podrían instalar un discurso despolitizado y excluyente, que aporta a clausurar en vez de promover un diálogo crítico.

El artículo presenta en un primer acápite las referencias teóricas que asumimos como base para comprender la heteronormatividad en la educación. Así nos adentramos en la comprensión de lo que es la intervención educativa y su relación con la heteronormatividad y el patriarcado. A continuación, se describe brevemente algunos antecedentes históricos sobre cómo desde las ciencias médicas y sociales a través de la historia, se ha contribuido al establecimiento de un imaginario excluyente y patologizador de la diversidad sexual. Posteriormente, desarrollamos una discusión respecto de los elementos heterosexistas que consideramos aún presentes en los discursos educativos actuales, contemplando nociones como la normalización, el heterocentrismo y la heteronormatividad, la despolitización del género, la apelación a una identidad fija, la noción de anormalidad y patologización, y el juego entre igualdad y diferencia.

\section{Claves teóricas: intervención educativa}

La intervención educativa corresponde a la búsqueda del "desarrollo integral" del/a estudiante por medio de una acción intencionada y planificada, considerándolo/a como un/a agente activo/a en su proceso formativo. Se busca un cambio de estado inducido por quien enseña, no sólo vinculado a los contenidos cognitivos o destrezas técnicas, sino también provocado por la reflexión sobre valores, actitudes, normas de convivencia, patrones culturales y ciudadanía, que son reflejadas en la vida cotidiana. Para llevar a cabo este proceso, es necesario que toda la comunidad educativa y los agentes de la

2 Esta comisión fue integrada por un psiquiatra infanto- juvenil, una doctora en psicología especialista en medicina familiar, el director del programa Iniciativa América del Hogar de Cristo y el vicepresidente de la Fundación para la Superación de la Pobreza (Palma, Reyes y Moreno, 2013). 
educación, como el Estado, estén involucrados en el proceso y se enmarquen en la consideración de la diversidad (Touriñán, 2011).

Sin embargo, muchas veces las intervenciones educativas que se centran en la diversidad como pilar principal, no manifiesta de forma clara, crítica y coherente qué están entendiendo por diversidad o cómo se está representando a la diversidad en las aulas. Consideramos que muchas veces la noción de diversidad puede operar invisibilizando diferencias y especificidades, además de mantener y promover representaciones patriarcales, heterosexistas y etnocéntricas.

Por ejemplo, la "Política Nacional de Convivencia Escolar 2015-2018" (Ministerio de Educación, 2015), si bien constituye un esfuerzo por mejorar la inclusión educativa de la diversidad en el aula, continúa explicitando que las "diferencias" son casos particulares y no parte de la diversidad humana. Al estar dirigido a un/a ideal de estudiante, a saber: heterosexual, "blanco", sin discapacidad y de clase media, materializa una lista de diferencias entre las que incluye: "necesidades educativas especiales de carácter transitorio (déficit atencional, hiperactividad, etc.) o carácter permanente (ceguera, sordera, etc.), diversidad de género, orientación sexual, diversidad generacional, política, socioeconómicas, cultural y étnica, etc." (p. 31).

Es así que consideramos que las miradas feministas que se rigen por el principio de la crítica y la sospecha (Lloyd, 2005), nos instan a estar atentas/os a los modos en los cuales las sociedades patriarcales se han transformado, asumiendo nuevas formas de control social relativas al género, las cuales muchas veces operan de modos menos evidentes y son por lo mismo más difíciles de desenmascarar, al haberse ido instalando con fuerza la "ilusión de vivir en un mundo de iguales" (Biglia, 2003, p. 2). Pero tampoco debemos negar que ha habido a su vez posibles avances y que aun cuando "la diversidad que se manifiesta hoy en la vida de las mujeres y en todo el mundo no debe confundirse con igualdad o justicia sexual o de género", también en ocasiones es su equivalente (Eisenstein, 2008, p. 18).

Es importante visualizar cómo en la intervención educativa emergen y toman fuerza ciertas construcciones sobre lo diverso, mientras otras quedan latentes o desaparecen. Dichas tramas se enmarcan en relaciones de poder que resultan convenientes para determinados órdenes sociales, a través de la producción de regímenes de inteligibilidad que legitiman ciertas verdades frente a otras. Este conjunto de prácticas y operaciones de gobierno permiten constituir, definir y organizar las poblaciones, de tal modo que no sea necesario recurrir al ejercicio propio de la fuerza y la coerción del paradigma jurídico para poder ejercer poder (Foucault, 1975). En ese sentido, la educación está traspasada por diversos mecanismos de gubernamentalidad, que inciden en la conducta de los individuos y colectivos; no solamente a través del control, la disciplina o la normalización, sino también por medios más sutiles (Rose, 1996).

En el caso de la diversidad sexual, hemos asistido históricamente a diversas formas de llamados a la normalización, a homogenizarse, a verse como el resto de la población: desde sistemas de represión directa (confinamientos o terapias de reconversión), o a través del encubrimiento de conductas y comportamientos considerados "afeminados" en hombres y "masculinos" en mujeres, imponiéndose la necesidad de coherencia entre sexo/género desde una mira normativa heterosexual. 
La intervención educativa entra de lleno en la lógica de la gubernamentalidad en el sentido que genera los mecanismos por los cuales se reproducen conocimientos y explicaciones sobre (y para) las vidas de las poblaciones, como el caso de las personas que se alejan del paradigma heteronormativo patriarcal, a partir de una posición institucional legitimada socialmente (educadores/as).

\subsection{Heteronorma y patriarcado}

En este artículo buscamos hacer una crítica a los marcos heteronormativos y patriarcales presentes en el ámbito de la educación, y sus efectos en el ámbito de las diversidades sexuales. Cuando hablamos de heteronormatividad estamos desnaturalizando la heterosexualidad, afirmando que ésta opera como un régimen político, compulsivo e institucionalizado que promueve relaciones de poder desiguales entre los géneros (Rich, 1980). Consideramos que los conceptos que usamos son claves, ya que nos permiten pensar de otros modos y construir otro tipo de realidades. En ese sentido hablar de "heteronormatividad" en vez de "homofobia" implica un cambio de enfoque y objeto de análisis importante, ya que se pasa de una mirada psicologicista, a una mirada más amplia, compleja y política que problematiza el estatus de normalidad y naturalidad de la heterosexualidad como matriz de ordenamiento social.

La heteronormatividad nos permite problematizar los modos en los cuales se estructura la sociedad a partir de un sistema binario (en el cual sólo tienen cabida hombres y mujeres), y jerárquico (de dominación masculina). La noción de heteronormatividad ha operado como una crítica importante al interior de los estudios feministas, y fue particularmente desde feminismos lésbicos que se han cuestionado conceptualizaciones tradicionales de la noción de género que reproducen un orden heterosexual (Butler, 2006, 2007). La heterosexualidad entendida como régimen político reconoce la imposición y la obligatoriedad de dicha norma, la cual es constantemente resistida, pero cuya desobediencia tiene muchas veces consecuencias que se traducen en diversas formas de discriminación (Guerra, 2009).

En las últimas décadas diferentes teóricas feministas y queer han planteado que la heterosexualidad predominante en las sociedades contemporáneas es un sistema de pensamiento omnipresente y que atraviesa todas las dimensiones de lo social (Butler, 2007; De Lauretis, 1987; Rubin, 1975; Wittig, 1992). Wittig (1992) en su clásico ensayo "El pensamiento heterosexual", enfatiza que la heterosexualidad es un pensamiento transversal a todas las ciencias que producen una cierta norma heterosexual en material de sexo, género y filiación. Esto llevaría a que se naturalice la heterosexualidad y permanezca como sustrato de los sistemas de pensamiento y por tanto, sea difícil no reproducir los supuestos básicos en los que se asienta: la diferencia sexual y la obligatoriedad de constituirnos en hombres y mujeres.

Sigue habiendo en el seno de esta cultura un núcleo de naturaleza que resiste al examen, una relación excluida de lo social en el análisis y que reviste un carácter de ineluctabilidad en la cultura como en la naturaleza: es la relación heterosexual. Yo la llamaría la relación obligatoria social entre el «hombre» y la «mujer». (...) Habiendo planteado como un saber, como un principio evidente, como un dato anterior a toda ciencia, la ineluctabilidad de esta relación, el pensamiento heterosexual se entrega a una interpretación totalizadora a la vez de la historia, de la realidad social, de la cultura, del lenguaje y de todos los fenómenos subjetivos. (Wittig, 1992, p. 51) 
Como hemos visto a través del tiempo, el campo de la sexualidad ha sido definido desde diferentes disciplinas y constreñido a una distinción entre las dicotomías: normal/anormal, y patológico/sano. Dirá Foucault (1977), que "el sexo no es cosa que sólo se juzgue, es cosa que se administra. (...) Policía del sexo: es decir, no el rigor de una prohibición sino la necesidad de reglamentar el sexo mediante discursos sutiles y públicos" (p. 34).

La crítica a la heteronormatividad permitió reconocer el papel central que la heterosexualidad juega en la mantención y naturalización de relaciones jerárquicas de género y la consecuente subordinación de las mujeres (Schilt y Westbrook, 2009). La noción de patriarcado apunta a su vez al reconocimiento de una estructuración dicotómica y jerárquica de las sociedades, en las cuales los hombres suelen poseer un estatus superior. Sin embargo, al igual que la mayoría de los conceptos centrales de teorizaciones feministas, la noción de patriarcado ha sido fuertemente contestada (Pease, 2014). Se ha cuestionado que muchas teorizaciones feministas al problematizar la violencia de género terminan retratando a hombres como intrínsecamente violentos, lo cual contribuiría a reproducir una dicotomía generizada de víctima/victimario y obstaculizaría las posibilidades de transformación de relaciones de género. Cuando se habla de violencia patriarcal se enfatizan las estructuras de poder que enmarcan las vidas de hombres y mujeres estableciendo jerarquías generizadas, reconociéndose los privilegios y posición de dominación que los hombres experimentan como grupo. Conceptualizaciones más recientes de patriarcado enfatizan a su vez la necesidad de visibilizar la complejidad de las relaciones patriarcales de poder en las cuales otras diferencias como las de ingresos económicos, étnicas y sexualidades no heteronormativas, entre otras, establecen relaciones de opresión entre hombres y grupos de hombres (Barker, 2016).

Podemos afirmar que la noción de patriarcado se ha vuelto más flexible y compleja, siendo cada vez más importante recalcar que no se trata de una estructura atemporal ni universal, sino de un sistema de poder que se articula de modos complejos con otros sistemas de control vigentes. Como veremos más adelante desde miradas feministas interseccionales es clave reconocer la articulación entre diferentes sistemas dominación y procesos de diferenciación. En esta línea se denuncia, por ejemplo, un "capitalismo heteropatriarcal racialmente estructurado" que a su vez demanda análisis conjuntos de los efectos del racismo, clasismo, sexismo y heteronorma (Hooks et al., 2004).

\subsection{Antecedentes históricos}

La discriminación hacia las personas LGTBI (lesbianas, gays, trans, bisexuales e intersex) está asentada en imaginarios simbólicos que tienen repertorios discursivos que no sólo son culturales, sino donde las organizaciones públicas, las ciencias en general y las ciencias sociales en particular, han incidido a partir de una actuación heteronormativa. Así, algunas voces "expertas" se revisten de "verdad" para regular la coherencia de la vida sexual y el deseo. Por ejemplo, la psicología, la psiquiatría, las actuaciones jurídicas, los marcos educativos o las perspectivas biológico-médicas han buscado una justificación a la diversidad sexual, enjuiciándola como un problema digno de ser investigado.

En esta lógica, el hecho de plantearse esta "problemática" implica comprender cualquier tipo de diversidad sexual que no se ajusta a los cánones heterosexistas, como un problema científico que perpetúa formas de discriminación de la diferencia. Estudios que intentan dar una "explicación" a la existencia de personas LGTBI como: la búsqueda de 
influencias neurohormonales durante la gestación (Ellis y Ames, 1987), la determinación de las estructuras neuroanatómicas para la explicación del comportamiento sexual (Zegers, Larraín y Bustamante, 2007) y la relación entre la proporción del dedo índice y del dedo anular entre hetero y homosexuales (Mustanski, Chivers y Bailey, 2005), no han hecho otra cosa que intentar explicar desde una mirada heteronormalizadora la diversidad humana, esta vez visibilizando la diferencia, pero desde una perspectiva patológica, curiosa o ajena a la "normalidad" del comportamiento humano.

Actualmente nuevas formas de coerción de la diversidad sexual desde las ciencias se desarrollan bajo otros fundamentos que buscan aparecer como éticamente correctos: este es el caso del desarrollo de terapias psicológicas de conversión justificándose a partir del supuesto malestar, la angustia y sufrimiento que algunas personas homosexuales manifiestan. En el caso chileno, existen ámbitos en la academia que actualmente desarrollan terapias de conversión ${ }^{3}$, basadas en fuertes componentes heteronormativos: prohibición de amistades homosexuales, reiteración del rol de género masculino clásico, promoción de actividades culturales sexistas, y terapia para enfrentar la pérdida de un padre ausente.

Estas actuaciones se ubican desde la dicotomía patriarcal, reduciendo las posibilidades de ambigüedad de sexos y géneros. Se apela, de esta manera, a que los sujetos se estructuren en una identidad "clara" y explícita, de manera de poder desarrollar las actuaciones pertinentes para favorecer su inclusión en relación a ese foco identitario explicitado.

La resistencia a estas actuaciones se expresa de diversa manera e históricamente hemos asistido a las luchas de los diversos grupos en pos de demandas concretas de reconocimiento y de derechos. Butler (2006) criticará que estas actuaciones siguen insistiendo en la lógica binaria. Sin embargo, también existen difracciones y puntos de resistencia en propuestas de grupos que subvierten las representaciones convencionales de la subjetividad sexual tradicional. Desde feminismos postestructuralistas se cuestiona el mismo concepto de género, en vías a romper las categorías y sus efectos reificantes, instalándose la noción de disidencia sexual (Echeverría y Maturana, 2015).

\section{Elementos heterosexistas aún presentes en los discursos educativos}

Desde el año 2015, el Ministerio de Educación de Chile (MINEDUC) implementó una política nacional de convivencia escolar, que busca garantizar un enfoque inclusivo y de derechos en los colegios y que se propicie la cultura escolar hacia el reconocimiento e incorporación de la diversidad en los procesos educativos. De acuerdo a esta política, el objetivo:

... es orientar la definición e implementación de acciones, iniciativas, programas y proyectos que promuevan y fomenten la comprensión y el desarrollo de una Convivencia Escolar participativa, inclusiva y democrática, con enfoque formativo,

\footnotetext{
${ }^{3}$ La psicóloga Marcela Ferrer, de la Universidad Católica de Chile, desarrolla actualmente un tipo de terapia de reparación para personas que son atraídas por otras de su mismo sexo. Su tesis de magíster se titula "Percepción infantil de no ser aceptado como un factor predisponente a la homosexualidad". Recuperado de http://www.bioeticacs.org/iceb/investigacion/4_Ferrer_Marcela_Prevencion_homosexualidad.pdf
} 
participativo, de derechos, equidad de género y de gestión institucional y territorial. (MINEDUC, 2015, p. 6)

No obstante, creemos importante explicitar algunos elementos naturalizadores y heterosexistas que pueden coexistir pese a la difusión de este enfoque inclusivo que no siempre es implementado por los establecimientos educativos ${ }^{4}$. En el fondo, buscamos aportar a visualizar qué representaciones funcionan como telón de fondo en dichas actuaciones. De esta manera, reflexionaremos sobre algunas tensiones que nos parecen centrales para pensar de manera crítica la noción de diversidad en general, y la diversidad sexual de manera particular en la intervención educativa.

\subsection{Normalización ciudadana e interseccionalidad}

En la educación, los discursos de derechos vinculados a la lógica de defensa a la igualdad, se relacionan con una aspiración de "aceptación" y de "integración social" de los sujetos LGTBI. Se generan exhortaciones de sensibilización respecto de su diferencia y la necesidad de que dispongan de las mismas oportunidades que el resto de la ciudadanía, considerada como heterosexual. Sin embargo, en estos procesos se invisibiliza la diferencia radical que establece el mismo sistema entre una supuesta sexualidad normal, la mayoritaria, y las diferentes (Sáez, 2004). La misma política nacional de convivencia escolar, reconoce en su diagnóstico que:

Necesidades educativas especiales de carácter transitorio (déficit atencional,
hiperactividad, etc.) o carácter permanente (ceguera, sordera, etc.), diversidad de
género, orientación sexual, diversidad generacional, política, socioeconómicas,
cultural y étnica, etc., son elementos constitutivos de la realidad que los
establecimientos educacionales ya tienen, pero que los abordan como problemas.
(MINEDUc, 2015, p. 31 )

Socialmente existen discursos que establecen la diversidad sexual como anormal dentro de una sociedad que considera a lo heterosexual como norma. En algunos ámbitos educativos se ha ido avanzando hacia una educación que posibilite un lugar al reconocimiento LGTBI. No obstante, pese a estas intenciones se sigue estableciendo esta diferenciación radical a partir de otra dicotomía: "mayoría/minorías". En esa distinción se reifica la distinción entre heterosexuales vinculados a la sexualidad regular, y quienes no tienen estas prácticas regulares, la comunidad LGTBI. Por tanto, vuelve a colocarse en esa distinción una carga negativa sobre lo que queda fuera del conjunto mayoritario. Lo que queda fuera, vuelve a considerarse por tanto, anormal. Así vemos que la heterosexualidad actúa como sistema político (Wittig, 1992/2006) que funciona transversalmente en todo el espectro social y también en el educativo que incide directamente en la construcción de los sujetos. Se puede visualizar como ejemplo como en las "Orientaciones para promover una escuela inclusiva" del MINEDUC (2013) cuando se plantea la necesidad de políticas de acción afirmativa, se plantea que éstas son para "la protección especial a las minorías" (MINEDUC, 2013, p. 10).

Asimismo, también se evidencian algunos silencios construidos en este campo, principalmente sobre la enunciación de las diversidades sexuales en relación con el género y la raza, así como con las diferencias de clase o de cultura étnica, de generación

${ }^{4}$ En el año 2012, el Ministerio de Educación informó que sólo el 12,15\% de los fondos destinados a capacitar a profesores en los programas de Educación Sexual se ejecutó durante ese año, participando sólo 221 recintos de los 467 que cumplían con los requisitos para optar al perfeccionamiento docente en sexualidad, afectividad y género. 
y de situación geográfica y sociopolítica (De Lauretis, 1991). De esta manera, generalmente se apela a una "diferencia" como una construcción aséptica que señala una distinción entre exo y endogrupo, y no se visualiza que nos encontramos con una diferencia jerarquizada, es decir, un problema relacionado con la posicionalidad y la asimetría (Anthias, 2002). A manera de ejemplo, podemos visualizar como el estudio desarrollado por el Instituto Nacional de la Juventud (INJUV) en 2014, "Percepciones en torno a la diversidad sexual", desarrolla un proceso de investigación centrándose especialmente en la diferencia sexual para poder ponderar la actitud de los/las jóvenes en Chile hacia los colectivos LGTBI. Sin embargo, no considera ninguna variable que cruce la diversidad sexual con otras marcas de diferencias que operan en los procesos de discriminación social, como son la clase, la etnia, la procedencia nacional. De esta manera la diferencia sexual aparece como un único vector de diferenciación, fácilmente superable por las nuevas generaciones sociales (INJUV, 2014). Por tanto, muchas veces, se invisibilizan otros ejes de diferenciación en el caso de la comunidad LGTBI al privilegiar por ejemplo la identidad sexual, dejando a un margen la posición socioeconómica de los sujetos.

Cabe señalar que esta metáfora de intersecciones entre factores de diferencia, no implica una sumatoria de posibles exclusiones y niveles identitarios, es decir, una adición de diferencias, sino más bien una conformación variable de significado que se activa contextual e históricamente. El establecimiento de las diferencias es así relacional y contextual que, a su vez, se articula con otras diferencias específicas. Es decir, una diferencia significativa o marca de opresión en un contexto determinado no es un atributo fijo y estable, sino una relación contingente y situada que se moviliza en cada práctica, esto es, atendiendo siempre a sus contextos de emergencia (Crenshaw, 1989).

En las intervenciones educativas aun cuando se trata de la sensibilización entorno a las vivencias LGTBI se parte de ciertas categorías sociales estandarizadas sobre las vivencias e identidades. En cambio, desde este punto de vista interseccional, no se conciben las categorías identitarias como algo esencial y pre-existente (LGTBI) -visión usual en los sistemas educativos- sino que se deconstruyen las categorías, poniendo el centro de interés en los regímenes de poder en que éstas son construidas, lo cual posibilita ciertas trayectorias de los sujetos. En el fondo, se trata de comprender cuándo y por qué estas categorías emergen como problemáticas y bajo qué factores. De esta manera, esas categorías constituidas a través de la diferencia, son producciones histórico-culturales con un claro ensamblaje semiótico-material. Por ejemplo, en el país existía un silencio frente a abusos basados en la orientación sexual o identidad de género de las personas, hasta el asesinato de Daniel Zamudio en el año 2012. A partir de ese evento, se generaron diversas directrices ministeriales para incluir el combate a la discriminación relacionada con la diversidad sexual y materiales educativos ad hoc. De esa forma la diversidad sexual se convirtió en objeto de atención, pero estructurada bajo discursos con un enfoque identitario centrado en la sexualidad, sin considerar otros factores como la clase o raza que inciden en los procesos de discriminación cotidiana ${ }^{5}$.

Asimismo, en este juego de categorías sociales, donde muchas veces se juega el reforzamiento identitario a partir de estos ejes de diferenciación, se invisibiliza 5 Ver "Educando sobre la diversidad" de MOviLh (2010), "Materiales sobre Diversidad sexual, prevención del suicidio y
Bulliying" de Todomejora (2012), Orientaciones generales para la atención de niños/as LGTB para profesionales de la salud" (2013), entre otros. 
realidades que pueden resultar fuera de la norma precisamente por la rigidez de esas categorías sociales vigentes. Así, hay sujetos sociales que nunca están presentes en la discusión: no tienen reconocimiento efectivo para ser considerados como sujetos en igualdad de derechos y como actores políticos. Como ejemplo cercano de este tipo de situaciones emerge el caso de la niña transexual que fue discriminada en el año 2015 por el colegio Pumahue, en la capital; entidad educativa que se negó a la solicitud realizada por el padre y la madre de la niña de ser tratada de acuerdo a su identidad de género.

Por otro lado, la feminista negra Patricia Hill Collins (2009) enfatiza que los diversos ejes de diferenciación están entrelazados en una "matriz de dominación" en la que se constituyen los sujetos en diferentes y cambiantes posiciones de poder. De esta manera, advierte que los sujetos oprimidos pueden incluso, dependiendo de algunas circunstancias y otros cruces, convertirse en opresores o reproductores de opresión a la vez. Para Hill Collins (2009) la matriz de dominación refiere a la organización general del poder en una sociedad, caracterizándose toda matriz de dominación por una articulación particular entre sistemas de opresión. Los modos en los cuales determinados sistemas de opresión intersectan dependerá de los contextos sociales e históricos específicos en los cuales se sitúan. Además, los sistemas de opresión intersectantes se organizan de modos específicos a través de cuatro dominios de poder interrelacionados: estructural, disciplinario, hegemónico e interpersonal.

\subsection{La despolitización de la noción de género}

Cuando se habla de adoptar una perspectiva de género, muchas veces pareciera asumirse que existe consenso respecto a lo que se está entendiendo por este concepto. Nos parece importante tener en cuenta que existen diversos modos de conceptualizar los géneros, y que no existe una forma única ni transhistórica de definirlos (García Fernández y Montenegro, 2014). Se trata por lo tanto de una categoría analítica que no existe con independencia de las teorías que la construyen de determinados modos siempre contextualizados y relativos a intereses específicos. La necesidad de repensar, debatir y cuestionar constantemente qué se está entendiendo por "género" y qué efectos genera, ha dado lugar a discusiones tremendamente enriquecedoras y transformadoras en el campo de los estudios feministas ${ }^{6}$.

La apropiación feminista de la noción de género ha sido central a la hora de cuestionar el determinismo biológico a partir del cual se asocian determinados roles, tareas y expectativas sociales a las personas en función de su genitalidad (Lykke, 2010). A pesar de las críticas posibilitadas por una definición tradicional de género, la dicotomía sexo/género ha sido a su vez ampliamente cuestionada desde diversos feminismos. Se cuestiona que la diferenciación tradicional entre sexo y género, reifica una mirada heteronormativa y dicotómica, a partir de la cual sólo son inteligibles hombres y mujeres.

Esta mirada se ha instalado con fuerza en políticas públicas, como las educativas, y a su vez en ámbitos académicos que no dialogan con estas otras miradas críticas feministas. Esta conceptualización hegemónica de la diferencia entre sexo y género ha sido cuestionada por perspectivas feministas postestructuralistas debido a que mantiene tanto

${ }^{6}$ Es así que, por ejemplo, a partir de la necesidad de reconocer la articulación de categorías de diferencia como el género, la raza y la clase promovida desde feminismos negros, socialistas y chicanos, entre otros, las teorizaciones en torno a la categoría género se fueron complejizando, dando lugar a perspectivas feministas interseccionales. 
una lógica heterosexista como una diferenciación dicotómica entre lo biológico y lo sociocultural, estableciéndose el cuerpo y la anatomía como ámbitos incuestionados (Butler, 2007; Fausto-Sterling, 2006; Sabsay, 2011). De este modo, al problematizarse solamente la institución social de los sexos, se establece una mirada culturalista del género que ha ido debilitando el potencial crítico feminista de desestabilización de un orden tradicional del género.

Es más, a pesar de la riqueza de los debates feministas en torno a la categoría género, la noción se ha despolitizado y desvinculado de las críticas feministas, operando muchas veces como sinónimo de sexo, al haberse establecido como el modo políticamente correcto para hablar de la diferencia sexual. Cuando la noción de género reemplaza sencillamente a la de sexo, se contribuye tanto a la mantención de un orden tradicional, binario y heterosexista basado en diferencias anatómicas naturalizadas, como a la reproducción de relaciones de dominación generizadas, lo cual limita y obstaculiza la apertura de espacios críticos y liberadores de fuga, desestabilización y problematización.

Por otro lado, también se presentan discursos basados en la catalogación de los sujetos desde una diferencia sexual binaria (sea ésta hombre-mujer o bien hetero/homo) dejando como ciudadanías abyectas a otras posibilidades de identificación sexual. Por ejemplo, las prácticas contra el bullying en la escuela, estimuladas desde la sociedad civil y el gobierno (ver, MINEDUC, 2014; Todomejora, 2013), suelen estar vinculadas a la lucha contra el acoso homosexual y lésbico, pero existen pocas prácticas visibles respecto de comunidades intersex, trans o queer.

La falta de representación y de visibilidad de la diversidad dentro de contextos normativos (como las escuelas), fomenta discursos homofóbicos y transfóbicos, bullying y acoso escolar. Así, esta reproducción de la heteronormatividad, genera espacios de inseguridad para estudiantes LGTBI (GLSEN, 2014).

Los casos de matonaje escolar, bullying o acoso escolar por identidad/orientación sexual, se corresponden con expresiones heterosexistas y patriarcales que menosprecian cualquier tipo de diversidad sexual. Como ejemplos podemos ver que en España el 65\% de chicos que se identifican como homosexuales han sido insultados (Vioque, Cascone y Martínez, 2013), mientras que un 84,6\% de las y los estudiantes de Estados Unidos señalaron que fueron insultadas/os en el último año por considerarse LGTBI (GLSEN, 2014). Estas prácticas implican “(a) la conducta agresiva y la intención de causar daño; (b) la reiteración en el tiempo, y (c) la ocurrencia en relaciones interpersonales y grupales en las que hay diferencia de poder" (Vioque, Cascone y Martínez, 2013, p. 847), esto potenciado por una masculinidad hegemónica que intenta imponer una heteronorma eliminando las posibilidades de la diversidad. Los elementos anteriores repercuten en la salud mental de las personas, generando una serie de consecuencias negativas y muchas veces llevándolas a suicidarse (GLSEN, 2014).

Otro ejemplo de educación planteada en términos dicotómicos la podemos encontrar en el Manual de Orientación de Educación Sexual del MINEDUC, "Orientaciones para el diseño e implementación de un programa en sexualidad, afectividad y género", donde se establece como concepto de género:

\footnotetext{
...los atributos sociales y a las oportunidades asociadas con el ser femenino $y$ masculino y las relaciones entre mujeres y hombres, como también entre las mujeres y entre los hombres. Estos atributos, oportunidades y relaciones están construidos socialmente y son aprendidos a través de socialización y varían según el contexto
} 
social y temporal. El género determina lo que puede esperarse, lo que es permitido y valorado en una mujer y un hombre en un contexto dado. (MINEDUC, 2014, p. 11)

\subsection{Dispositivos heterocentrados}

El heterosexismo ha establecido que las únicas formas válidas de sexualidad son las que se puedan establecer entre mujeres y hombres, sin hacer distinciones o presentar matices entre diversas identidades sexuales o de género (Welzer-Lang, 2002). Desde esta posición, la intervención educativa ha adscrito a la categoría subalterna de "los diversos" para clasificar a las personas LGTBI o con identidades sexuales o de género que no se condicen que la heteronormatividad. Bajo la idea de "los/as otros/as" o "diferentes" se ha buscado "integrar" y "comprender" identidades no normativas presentes en el contexto escolar (Echeverría y Maturana, 2015), principalmente por motivaciones que se vinculan con la expresión de violencia contra los y las que está "fuera de la norma".

Independientemente de las intenciones, el binarismo "heterosexual/homosexual" ha operado en esta lógica, como si fueran polos opuestos del comportamiento humano o como formas excluyentes entre sí, imponiéndose un ideal de heterosexualidad como primario, acrítico y "deseable" (Esguerra y Bello, 2014). Esta heteronormatividad impregna las conceptualizaciones de género, presentes en la institución pública y educativa (Echeverría y Maturana, 2015), lo que legitima la discriminación, retroalimentando las acciones que definen a "los diversos" como la otredad.

Un ejemplo de esto, es que en muchos textos escolares chilenos de enseñanza básica, perdura la idea de "la familia feliz heterosexual" como única forma de constitución familiar lo que es un reflejo de la concepción de familia presente desde las políticas públicas (Marchant, Soto y López, 2009). Y aunque "los/as diversos/as", que no adscriben a este ideal de familia, podrían adoptar formas similares (pero no iguales bajo la ley) para conformar una familia, a través del reciente Acuerdo de Unión Civil ${ }^{7}$, esto se invisibiliza primando un proceso de socialización que se centra en la heterosexualidad.

De esta manera, el ámbito de la educación funciona como un dispositivo heterocentrado ya que bajo la adopción de un "nosotros/as los/as semejantes" se invita a integrar "lo diferente", sin asumir las propias diferencias dentro de los grupos heterosexuales. Así, los niños y niñas LGTBI quedan supeditados a una inclusión que viene de un sector heterosexual -con lo cual quedan invisiblizados como agentes- ya que este discurso de inclusión se dirige hacia un "igual heterosexual" que es quien debe o es responsable de incluir (Echeverría y Maturana, 2015). Se confirma así lo que ya han señalado estudios anteriores (Echeverría y Maturana, 2015; Esguerra y Bello, 2014), al indicar la permanencia de marcos heteronormativos en las políticas públicas en pro de la diversidad sexual.

Junto con lo anterior, los procesos de socialización sexistas fomentan la discriminación y el silenciamiento de identidades no heterosexuales. Por ejemplo, existe una omisión en la sexualidad de personajes famosos o modelos de referencia, cuyas orientaciones e identidades (de forma explícita) sexuales son distintas de las heterosexuales (por ejemplo, en Chile: Gabriela Mistral), lo que implica una carencia en fuentes de información y apoyo, ausencia de referentes, sensación en las y los estudiantes de que

\footnotetext{
${ }^{7}$ La Ley de Acuerdo de Unión Civil fue promulgada el 13 de Abril del año 2015. Busca regular la desprotección y discriminación de las parejas que conviven, sean de igual o distinto sexo (Gobierno de Chile, 2015). Sin embargo, esto constituye una paradoja al no considerar filiación.
} 
una identidad/orientación sexual diferente a la heteronormativa es algo "extraño" o negativo, lo que conlleva a situaciones de aislamiento y silencio, o a la construcción de una propia identidad desde una perspectiva castigadora o culposa (Dorado, 2008).

Como vemos, se viven tensiones entre significados relativos a la igualdad y la diferencia. Se visualizan discursos que promueven la invisibilización de diferencias y de procesos estructurales históricos y sociales de dominación a través de la apelación a la igualdad o una humanidad compartida. En tiempos en los cuales las demandas feministas y de disidencias sexuales parecen estar tomando fuerza y mayor visibilidad, las respuestas de rechazo a estas demandas aparecen a su vez con mayor insistencia. En relación a esto mismo hemos visto en redes sociales como ante denuncias de sexismo, racismo y homofobia se apela muchas veces a existencia de discriminaciones inversas, lo cual ha dado lugar a numerosas discusiones en torno a si acaso la violencia que sufren los hombres no es violencia de género, si los heterosexuales no deberían ser también parte de la diversidad sexual o si acaso los negros no discriminan también a los blancos.

¿Por qué parece incomodar aún a muchas personas la idea de diferencia, y sin embargo las nociones de igualdad y diversidad parecen haberse instalado como intrínsecamente buenas/benevolentes? La noción de diversidad puede operar muchas veces de manera paradójica ya que invisibiliza las diferencias que supuestamente nombra e incluye. Debemos estar atentos/as a los usos de la noción de diversidad, y ser capaces de cuestionar cuando ésta obstaculiza abordajes críticos de las diferencias que nos constituyen y a partir de las cuales nos relacionamos. Así mismo es necesario distinguir entre diferentes usos de la "diferencia", para reconocer cuando ésta opera como un modo de protesta o resistencia frente a formas de opresión y explotación, y cuando la diferencia es invocada para operar como forma legitimación de relaciones de dominación (Brah, 2011).

Desde una mirada interseccional se nos insta a estar más atentos/as a los modos en los cuales operan y se materializan procesos de dominación internos al conjunto de diversidad sexual: así quienes se asimilan más al sistema imperante (heteropatriarcal capitalista colonial) pueden llegar a disfrutar de ciertos privilegios respecto a otros grupos cuyas luchas se ubican también en ámbitos de las sexualidades no normativas. El mejor ejemplo de esto, en el último tiempo, es ver como se ha posicionado con rapidez la defensa del matrimonio igualitario, pero no así la lucha a favor del aborto. Teniendo en cuenta las sociedades heteropatriarcales y capitalistas que habitamos, no nos debe extrañar que las demandas de "cierta" diversidad sexual han avanzado con mayor rapidez, ya que se ajustan a valores tradicionales y hegemónicos en un sistema capitalista que ha abierto las puertas al denominado "mercado rosa".

Es así que las políticas de reconocimiento de la diversidad sexual y de la equidad de género participan a su vez de procesos de rearticulación de jerarquías sociosexuales, instalándose nuevas homonormatividades en las cuales la familia y la pareja siguen operando como modelos hegemónicos de organización social (Sabsay, 2011). En el contexto neoliberal que habitamos las demandas de diversidad sexual que apelan a un modelo de familia y amor romántico tradicional, modelo que ha sido ampliamente cuestionado desde diversos posicionamientos feministas al constituir un elemento clave del sistema heteropatriarcal y capitalista, parecen adaptarse e integrarse con aparente facilidad (no por eso sin resistencias por parte de ciertos sectores más conservadores de 
la sociedad). Cabe preguntarse quiénes son representativos de esta diversidad sexual que ha logrado integrarse, y quiénes continúan siendo sistemáticamente excluidos/as.

\subsection{Apelación a una identidad fija y esencialista}

La diversidad sexual, entendida como reconocimiento de LGTBI, opera mediante la categorización de sujetos diversos en función de su orientación sexual (lesbianas, gays, bisexuales), su identidad de género (trans) o su sexo biológico (intersex). Aun cuando dichas categorías identitarias permiten construir posibilidades no heteronormadas de ser, desear e identificarse, terminan a su vez estableciendo etiquetas y categorizaciones que reproducen relaciones de inclusión/exclusión, construyendo normativas que establecen quienes formarían parte de cada una de estas categorías. De este modo se van construyendo tipos de sujetos estables, ampliándose el abanico de posibilidades, pero sin generar críticas más radicales a la noción de sujeto e identidad subyacente.

Por ejemplo, consideremos el uso de la categoría "gay" en discursos peyorativos que operan en ciertos contextos educativos con el objeto de denostar a las personas (GLSEN, 2014). Por lo general, su uso implica la delimitación de tareas y actividades vinculadas a un comportamiento esperado según la lectura social de un sexo/género particular, lo que, junto con otro tipo de prácticas discriminatorias, genera la exclusión o autoexclusión en la participación de actividades que son asignadas a géneros/sexos predeterminados (Avillo et al., 2015). Así, personas que sientan interés por una u otra actividad someterán su decisión a lógicas heteronormativas que pasarían a llevar sus intereses, lo que implica excluirse de determinados grupos y realizar las ocupaciones que se corresponden con su sexo/género.

Los Feminismos postestructuralistas han desarrollado importantes críticas a abordajes feministas que operan asumiendo un sujeto preexistente a sus políticas identitarias, enfatizándose la necesidad de un anti-esencialismo más radical. De este modo se problematizaron las maneras en las cuales se reifican diferencias entre hombres y mujeres en investigaciones feministas cuyo foco en la diferencia de género no deja ver cómo el género es simbolizado y producido (Leavy, 2006). Desde estas miradas no debe entenderse el género como algo que poseemos, sin desafiarlo, ya que así se terminarían fijando las diferencias de género y sexo sin aportar a su cuestionamiento y desestabilización (Cosgrove, 2002, en Leavy, 2006). No debemos perder de vista que la dominación opera justamente mediante la regulación y producción de sujetos (Leavy, 2006).

Consideremos por ejemplo el acto de "salir del closet" (Sedgwick, 1990 en Lloyd, 2005). Salir del closet es vivido por muchos/as como el acto de asumir y hacer pública una verdad interna acerca de uno/a mismo/a. Este "descubrimiento" implica a su vez una relectura y reescritura de la propia biografía en función de esta nueva verdad interior. Tal como establece Moya Lloyd (2005), salir del closet es algo más que la divulgación de un secreto íntimo, ya que implica la reconstrucción del pasado a la luz de esta nueva verdad revelada. En una sociedad en la cual las identidades sexuales se construyen como estables, verdaderas e inevitables, permeando todos los ámbitos de nuestro ser, salir del closet nos obliga a practicar una resignificación que de sentido a esta recientemente asumida "verdadera identidad". Todo aquello que permita dar coherencia biográfica a esta revelación será re-significado con ese fin. De este modo un asumirse lesbiana a una determinada edad puede implicar leer ciertos recuerdos a la luz de lo que se reconoce como auténticamente lésbico, por ejemplo, el no haber disfrutado jugar con muñecas, 
pero sí con camiones, no haberse maquillado o depilado y haber disfrutado jugar con autos y barro. Para Shane Phelan (en Lloyd, 2005) asumir una identidad lésbica es un acto performativo, no una constatación, la subjetividad lésbica es el producto de prácticas y discursos que la nombran, de comunidades históricas o discursivas de las cuales participamos. Estas comunidades históricas y discursivas constituyen los marcos de inteligibilidad que promuevan la construcción de biografías coherentes con una identidad sexual asumida como auténtica ${ }^{8}$.

No queremos poner en duda que muchas personas viven sus identidades sexuales a partir de un sentido de coherencia, estabilidad y continuidad, pero tampoco podemos desconocer que muchas personas no las viven de este modo. Es decir, hay personas que sienten que nacieron homosexuales y consideran que esta orientación sexual es parte de su naturaleza estable. Pero otras personas experimentan cambios en sus deseos, identidades, cuerpos y relaciones a lo largo de su vida, las cuales pasan a ser ininteligibles desde marcos normativos que apelan a identidades fijas y preexistentes.

Queremos apelar a la necesidad de un trabajo crítico constante, que implica un cuestionamiento y una problematización continua de saberes, prácticas, imaginarios sociales, y discursos que se vuelven hegemónicos en un contexto histórico particular. Judith Butler (2006) entiende por crítica:

Un cuestionamiento de los términos que restringen la vida con el objetivo de abrir posibilidades de modos diferentes de vida; en otras palabras, no para celebrar la diferencia en sí misma, sino para establecer condiciones más incluyentes que cobijen y mantengan la vida que se resiste a los modelos de asimilación. (p. 17)

Se trata por lo tanto de una crítica con fines políticos de transformación social, que permitan construir otras formas de vida, otras realidades y relaciones posibles en contextos sociales sistemáticamente violentos y opresores. Dicha actitud crítica debe ser constante, para constituirse en un desacuerdo con aquello que ya existe, un rechazo a lo que es insatisfactorio en el presente, ya que esto posibilita a los feminismos constituirse en un proyecto de oposición radical, inherentemente críticos y políticos (Lloyd, 2005).

\section{Conclusiones}

A través de la discusión anterior podemos ver que en el ámbito educativo se sigue construyendo a las personas LGTBI bajo identidades irreductibles y por tanto, desde una lógica de atención a las minorías, donde las diferencias en el grupo mayoritario heterosexual son invisibilizadas. Para construir esta otredad irreductible de las personas LGTBI, se evidencia un mecanismo doble: de diferenciación radical, por un lado, y de establecimiento de categorizaciones fijas. Esto es, una operación de diferenciación que se hace a través de mecanismos jerarquizados, en los cuales la figura de la diversidad sexual se entiende como diferente respecto de una supuesta comunidad de iguales -conformada por personas heterosexuales-, y en relación de asimetría respecto de los miembros del grupo mayoritario. Se genera de esta manera un proceso de subalternización (Dube, 2001), lo que conlleva que los sujetos sean posicionados en los márgenes del sistema social y que sean conceptualizados y representados desde el discurso de la otredad, y

${ }^{8}$ En Chile, se ha integrado en la resolución que fija el calendario escolar 2016 para los establecimientos educacionales, la celebración del Día Internacional contra la Homofobia y la Transfobia (17 de mayo), que corresponde al día en que la Organización Mundial de la Salud dejó de considerar a la homosexualidad como una enfermedad en 1990. 
desde una posición hegemónica. De esta manera los dispositivos de intervención educativa reproducen discursos que consideran a sus sujetos como carentes o deficitarios, limitando sus posibilidades de agencia.

Así cuando se apela a la diversidad sexual, son los otros y las otras, quienes están fuera de la norma, y el mundo mayoritario heterosexual quienes deben comprender esa diferencia. Por tanto, en el intento de incluir, nominativamente se excluye. En la pretensión de incluir la diferencia, se sitúa a lo diverso desde una otredad irreductible, ya que no se logra instalar la idea de un nosotros/as que incluya lo diverso.

Sin embargo, en ocasiones aparecen ciertas referencias a las diversidades sexuales, pero estas son consideradas como minorías. Considerar a las personas LGTBI desde esta perspectiva, sólo reifica la construcción de un sujeto en subordinación hegemónica, que además son precarizados. Por tanto, en contextos educativos a veces aparece reconocida sólo una parte del espectro sexual, dejando menos representados a quienes son disidentes de las lógicas más normativas. Quienes logran caber dentro de los espacios que permite la heteronormatividad, hacen más uso de estas posibilidades. De esta manera, muchas veces en las actuaciones se cuelan normalizaciones hegemónicas, que son las que precisamente se combaten.

Es importante tener en claro que esta lectura esbozada no es una apuesta evaluadora de las actuaciones y políticas educativas vigentes que han ido avanzando en ciertos grados de reconocimiento. Sino más bien como señala Bourdieu (2000), tener una cierta "vigilancia epistemológica" de nuestro accionar y cómo incluso pretendiendo una lucha de reconocimiento, a veces estamos actuando con nuestras prácticas y discursos, reificando unas diferencias excluyentes. Entonces cabe preguntarse si es posible la generación de una educación en pro de la diversidad sexual que sea emancipatoria, que rompa la lógica heteronormativa y dualista vigente o si se puede luchar por un reconocimiento cuando a la vez se lucha identitariamente. En ese sentido, los debates feministas actuales nos orientan y nos permiten estar alertas ante el levantamiento de nuevas categorías estancas y cerradas.

\section{Referencias}

Anthias, F. (2002). Beyond feminism and multiculturalism: locating difference and the politics of location. Women's Studies International Forum, 25(3), 275-286.

Avillo, C., Barrientos, B., Fernández, J., Matus, C., Olivares, C. y Morrison, R. (2015). Contexto y elecciones ocupacionales de adolescentes que se identifican como homosexuales. Revista Chilena de Terapia Ocupacional, 15(2), 34-51. doi:10.5354/0717-5346.2015.38157

Barker, G. (2016). Male violence or patriarchal violence? Global trends in men and violence. Revista Latinoamericana Sexualidad, Salud y Sociedad, 22, 316-330.

Biglia, B. (2003). Transformando dinámicas generizadas: propuestas de activistas de movimientos sociales mixtos. Athenea Digital, 4, 1-25.

Bourdieu, P. (2000). Intelectuales, politica y poder. Buenos Aires: EUDEBA.

Brah, A. (2011). Cartografías de la diáspora. Identidades en cuestión. Madrid: Traficantes de sueños.

Butler, J. (2006). Deshacer el género. Barcelona: Paidós.

Butler, J. (2007). El género en disputa. Barcelona: Paidós. 
Crenshaw, K. (1989). Demarginalizing the intersection of race and sex: a black feminist critique of antidiscrimination doctrine, feminist theory and antiracist politics. Chicago, IL: University of Chicago Legal Forum.

De Lauretis, T. (1987). Tecnologías del género. En T. De Lauretis (Dir.), Diferencias (pp. 33-69). Madrid: Ed. Horas y Horas.

De Lauretis, T. (1991). Queer theory. Lesbian and gay sexualities. An introduction. Journal of Feminist Cultural Studies, 3(2), 3-18.

Dorado, A. (2008). Desenmascarar el pensamiento heteronormativo de la educación en la diversidad afectivo-sexual: guías didácticas en Cataluña. Una óptica psicosocial crítica (Tesis doctoral). Universitat de Girona. España.

Dorlin, E. (2009). Sexo, género y sexualidades. Introducción a la teoría feminista. Buenos Aires: Nueva Visión.

Dube, S. (2001). Sujetos subalternos. México: Colegio de México.

Echeverría, G. y Maturana, J. M. (2015). Análisis crítico del discurso de políticas públicas en diversidad sexual en Chile. Universitas Psychologica, 14(4), 1485-1498. doi:10.1 1144/Javeriana.up 14-4.acdp

Eisenstein, Z. (2008). Señuelos sexuales. Género, raza y guerra en la democracia imperial. Barcelona: Edicions Bellaterra.

Ellis, L. y Ames, M. A. (1987). Neurohormonal functioning and sexual orientation: a theory of homosexuality-heterosexuality. Psychological Bulletin, 101(2), 238-258.

Esguerra, C. y Bello, J. (2014). Interseccionalidad y políticas públicas LGBTI en Colombia: usos y desplazamientos de una noción crítica. Revista de Estudios Sociales, 49, 19-32. doi: $10.7440 /$ res49.2014.02

Fausto-Sterling, A. (2006). Cuerpos sexuados. La política de género y la construcción de la sexualidad. Barcelona: Melusina.

Foucault. M. (1975). Defender la sociedad. Buenos Aires: FCE.

Foucault, M. (1977) Historia de la sexualidad I. La voluntad de saber. México: Siglo XXI.

García Fernández, N. y Montenegro, M. (2014). Re/pensar las producciones narrativas como propuesta metodológica feminista: experiencias de investigación en torno al amor romántico. Revista Athenea Digital, 14(4), 63-88.

GLSEN. (2014). Kit de espacio seguro: guía para ser un/a aliado/a de estudiantes LGBT. Santiago: Fundación Todo Mejora.

Gobierno de Chile. (2015). Preguntas y respuestas sobre la ley de unión civil. Recuperado de http://www.gob.cl/2015/02/04/acuerdo-de-union-civil-preguntas-y-respuestas-sobre-laproteccion-y-los-beneficios/

Guerra, L. A. (2009). Familia y heteronormatividad. Revista Argentina de Estudios de Juventud, 1, $1-17$.

Hill Collins, P. (2009) Black feminist thought. Knowledge, consciousness, and the politics of empowerment. Londres: Routledge.

Hooks, B., Brah, A., Sandoval, C., Anzaldúa, G., Levins Morales, A, Bhavnani, K., Coulson, M., Alexander, M. y Talpade, C. (2004). Otras inapropiables. Feminismos desde las fronteras. Madrid: Traficantes de sueños.

INJUV. (2014). Percepciones entorno a la diferencia sexual. Recuperado de http://www.injuv.gob.cl/ 
Leavy, P. (2006). Feminist postmodernism and poststructuralism. En S. N. Hesse Biber y P. Leavy (Comps.), Feminist research practice. A primer (pp. 83-108). Londres: Sage.

Lloyd, M. (2005). Beyond identity politics. Feminism, power and politics. Londres: Sage.

Lykke, N. (2010). Feminist studies: a guide to intersectional theory, methodology and writing. Nueva York: Routledge.

Marchant, J. P., Soto, E. y López, A. (2009). Teoría de género, feminismo y sus implicancias para la educación. Recuperado de http://mazinger.sisib.uchile.cl/doctos/teoria-genero.pdf

MINEDUC. (2013). Orientaciones para promover una escuela inclusiva. Santiago: Ministerio de Educación, Gobierno de Chile.

MINEDUC. (2014). Educación en sexualidad, afectividad y género. Orientaciones para el diseño e implementación de un programa en sexualidad, afectividad y género. Santiago: Ministerio de Educación, Gobierno de Chile.

MINEDUC. (2015). Política nacional de convivencia escolar. Santiago: Ministerio de Educación, Gobierno de Chile.

MINSAL. (2010). Ley $N^{\circ}$ 20.418. Santiago: Diario Oficial de la República de Chile.

Morgade, G. (2006). Educación en la sexualidad desde el enfoque de género. Una antigua deuda de la escuela. Novedades Educativas, 184, 40-44.

Morgade, G. (2011). Toda educación es sexual. Hacia una educación sexuada justa. Buenos Aires: La Crujía.

Mustanski, B., Dupree, M. N., Bocklandt, S. \& Schork, N. H. (2005). A genomewide scan of male sexual orientation. Human Genetics, $116(4)$, 272-278. doi: 10.1007/s00439-004-1241-4

Palma, I., Reyes, D. y Moreno, C. (2013). Educación sexual en Chile: pluralismo y libertad de elección que esconde que esconde una propuesta gubernamental conservadora. Docencia, $45,14-24$

Pease, B. (2014). Theorizing men's violence prevention policies. Limitations and possibilities of interventions in a patriarchal state. En H. Powell (Ed.), Preventing sexual violence. Interdisciplinary approaches to overcoming a rape culture (pp. 67-99). Nueva York: Palgrave Macmillan.

Rich, A. (1980). Compulsory heterosexuality and lesbian existence. Signs: Journal of Women in Culture and Society, 5(4), 631-660.

Rose, N. (1996). Inventing ourselves: psychology, power and personhood. Nueva York: Cambridge University Press.

Rubin, G. (1975). El tráfico de mujeres: notas sobre la economía política del sexo. Revista Nueva Antropología, 30, 95-145.

Sabsay, L. (2011). Fronteras sexuales. Espacio urbano, cuerpos y ciudadanía. Buenos Aires: Paidós.

Sáez, J. (2004). Teoría queer y psicoanálisis. Madrid: Síntesis.

Schilt, K. y Westbrook, L. (2009). Doing gender, doing heteronormativity: "gender normals", transgender people, and the social maintenance of heterosexuality. Gender and Society, $23(4), 440-464$.

Todo Mejora. (2012). Materiales sobre Diversidad sexual, prevención del suicidio y Bulliying. Fundación Todo Mejora [Página web]. Disponible en: http://todomejora.org/wpcontent/uploads/2015/10/TM-Material-Completo-sobre-diversidad-sexualprevenci\%C3\%B3n-del-suicidio-y-bullying-homof\%C3\%B3bico.pdf 
Todo Mejora. (2013). Orientaciones generales para la atención de niños/as LGTB para profesionales de la salud. Fundación Todo Mejora [Página web]. Disponible en: https://todomejora.org/wp-content/uploads/2015/10/Guia-TM-2-logo-nuevo.pdf

Touriñan, J. M. (2011). Intervención educativa, intervención pedagógica y educación: La mirada pedagógica. Revista portuguesa de pedagogía, 283-307.

Valdés, T., Sívori, H., Pezoa, S., Barreto, A., Rohden, F., Carrara, S., Heilborn, M. y Araujo, L. (2011). Género y sexualidad: competencias para la docencia. Santiago: CEDEM.

Vioque, S. B., Cascone, M. y Martínez, C. (2013). Estigma del sistema de género: aprendizaje de los modelos normativos, bullying y estrategias de resiliencia. Política y Sociedad, 5o(3), 837-864. doi:10.5209/rev_poso.2013.v50.n3.41971

Welzer-Lang, D. (2002). La crisis de las masculinidades: entre cuestionamientos feministas y críticas contra el heterosexismo. Recuperado de http://www.jerez.es/fileadmin/

Wittig, M. (1992/2006). No se nace mujer. En El pensamiento heterosexual y otros ensayos. Madrid: Ed. Egales.

Zegers, B., Larraín, M. E. y Bustamante, F. (2007). Sobre la homosexualidad. Santiago: Mediterráneo.

\section{Breve CV de los autores}

\section{Caterine Galaz}

Académica de la Universidad de Chile. Coordinadora del Núcleo de I+D "Estudios críticos de la diversidad" de Trabajo Social, de la Universidad de Chile. Doctora en Ciencias de la Educación, Universidad Autónoma de Barcelona. Email: cgalazvalderrama@uchile.cl

\section{Lelya Troncoso}

Psicóloga social feminista. Docente de la Universidad de Chile y la Universidad Diego Portales. Master en Psicología Social y Master en estudios de género. Doctora (C en Psicología, Universidad de Chile. Email: lelya.troncoso@gmail.com

\section{Rodolfo Morrison}

Doctor y Máster en Lógica y Filosofía de la Ciencia, Universidad de Salamanca. Académico y coordinador del área de investigación, Departamento de Terapia Ocupacional y Ciencia de la Ocupación, Facultad de Medicina, Universidad de Chile. Email: rodolfomorrison@med.uchile.cl 